\title{
Applied research on virtual reality technology in digital display design
}

\author{
Junzhu Zhang ${ }^{1, a}$, Bo Yin ${ }^{2, b}$,Yufu Chen ${ }^{3, c}$ \\ ${ }^{1}$ Shunde Polytechnic, Foshan, Guangdong, China \\ ${ }^{2}$ Guangdong Industry Technical College, Guangzhou, Guangdong, China \\ ${ }^{3}$ YunTech, Taiwan, China \\ ${ }^{a}$ email, ${ }^{b}$ email, ${ }^{c}$ email
}

Keywords: virtual reality technology; digital display; $B P$ neural network; evaluation model

Based on the theory of $B P$ neural network, this paper evaluates the simulation and observability of the virtual reality technology in the digital display design. Meanwhile, through a large number of questionnaire surveys, this paper collects the relevant data and establishes an applied evaluation model of the virtual reality technology in the digital display design. This paper selects Mount Huangshan, Mount Taishan, the Forbidden City and Wuzhen Town as research objects to verify the model. The evaluation results show that, the digital display effect of Mount Huangshan and the Forbidden City is good, but the digital display effect of Mount Taishan and Wuzhen Town is relatively poor. This conclusion is consistent with the actual situation, thus indicating the reasonability of the model.

\section{Introduction}

With constant updating of high-tech means, the digital display gradually becomes a platform for people to enjoy travel.It makes Internet, mobile terminal or display terminal as the carrier and draws three-dimensional images through the virtual reality technology so that the viewers can enjoy a strong visual impact. Under normal circumstances, the digital display design of the virtual reality technology is applied for virtual tourism, architectural design and other aspects. In terms of virtual tourism, the digital display design consists of multiple steps.First, examine field data and build scenes on the two-dimensional plane; then build scenes on the three-dimensional space by using 3DMAX modeling software and ${ }_{M a p G I S}$; finally, achieve the function of navigation maps and three-dimensional visualization in the virtual scene via the virtual tourism system on the integrated development platform of MapGIS data center. Currently,in terms of the architectural design, it mainly applies VRML to define a certain layout in the three-dimensional spaceand also presentthe three-dimensional effect of architectural design. In addition, Multi Creator and other professional software can be used to meet a higher display requirement on the sound effect and flows and so on. No matter the virtual reality technology is applied for the virtual tourism or architectural design, its digital display design effect is always the focus of attention.

Based on the mathematical theory of BP neural network, this paper focuses on the research of the simulation and observability of the virtual reality technology in the digital display design through analysis of the application effect of the virtual reality technology in the digital display design, and aimed at the establishment of an evaluation model for the application effect of the virtual reality technology in the digital display design.

\section{Virtual Reality Technology}

The virtual reality technology sets up a kind of three-dimensional virtual environment with the carrier of computer software and hardware resources. The users can immerse themselves in a virtual environment and experience the true feeling of the virtual environment according to their own wishes to operate the computer.

According to the different means of generating the three-dimensional scene, the virtual reality technology is divided into two types: the image-based virtual reality technology and the geometric modeling-based virtual reality technology. The former has a certain advantage in reflecting the 
authenticity of the landscape, while the latter requires a lot of computer calculation process. Therefore, it has a relatively high requirement on the computer. Currently, the virtual reality technology is used in many aspects such as the virtual tourism platform, real estate sales and exhibition and so on.

\section{Evaluation Of Digital Display Effect}

This paper will evaluate the display effect of multiple digital display platforms from the simulation and observability of the virtual reality technology in the digital display design by using $B P$ neural network model.

After consulting a large number of pertinent literatures, the "virtual Sweden" website is found to be a good digital display platformat present. This paper adopts the stratified random sampling method and selects 100 viewers, including college students, interior designers, managers of the real estate display platform and other random personnel to score the simulation and observability of the digital display with a total of 10 scores, and disposes the survey results (taking mean value for the score of crowd at each stage), thus we obtain Figure 1:

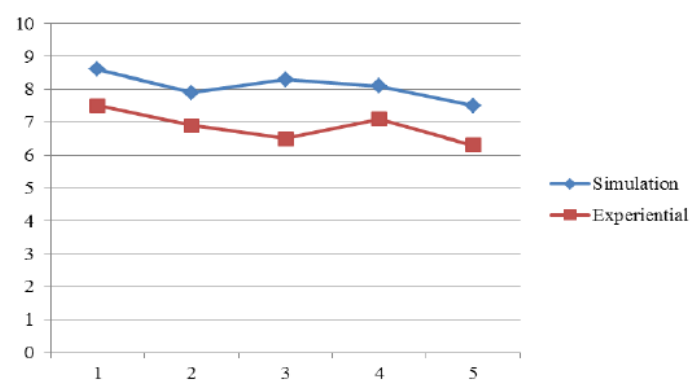

Figure 1. Eigenvalues distribution for excellent digital platform

The poor digital display platform is free of statistics. Therefore, during the questionnaire survey, the respondents can fill in the poor digital display platform according to their own understanding of the situation, and score for the simulation and observability of the website. The survey method adopted is the same with the survey method of the eigenvalues for excellent digital platform. The results are shown in Figure 2:

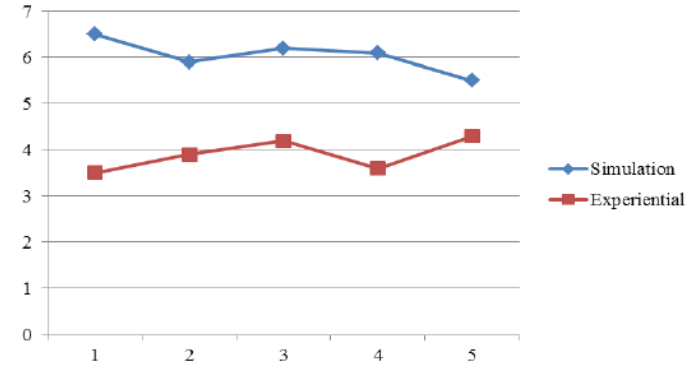

Figure 2. Eigenvalues distribution for poor digital platform

Select the digital display platforms in four places-(1)Shandong Yikang Elderly Health Industrial Park, (2)Shijiazhuang Office Building, (3)Zichuan Shanshuiyuan Community and (4)Wuzhen Town scenic spot as the research objects, and randomly select fifty respondents at the local place of the research objects. The respondents can evaluate four digital display platforms and dispose the survey results as mean values, thus we obtain Figure 3: 


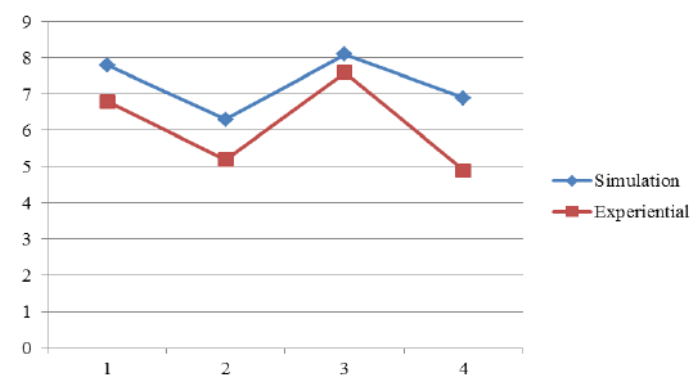

Figure 3. Eigenvalues distribution for digital platform

The neural network model originates from the neurobiology. Its calculation process is similar to the neuronal reaction process in the biology, as shown in Figure 4:

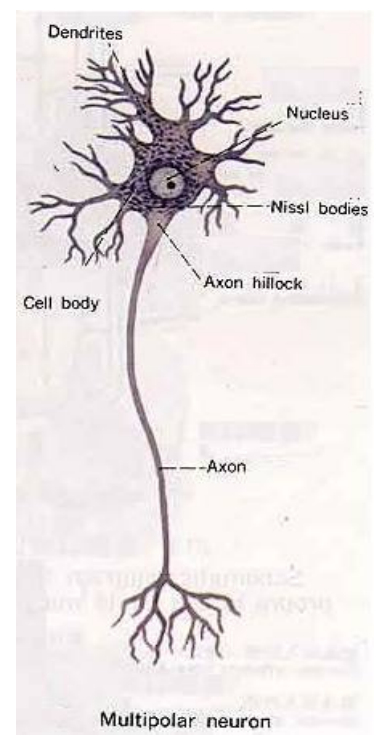

Figure 4.Structure of neurons

In the neural network, many different neurons, including axon terminals can enter the same neuron dendrite and form a large number of synapses. The neurotransmitter released by different sources of synapses can play a role in the membrane potential changes of the same neuron. As can be seen that, the neurons have the ability to integrate information, that is, the neurons can integrate different sources of input information on the dendrites. Based on this ability, the reaction process of the simulated neurons creates an artificial neuron model. From the perspective of the mathematical symbols, the expression is shown in Figure 5:

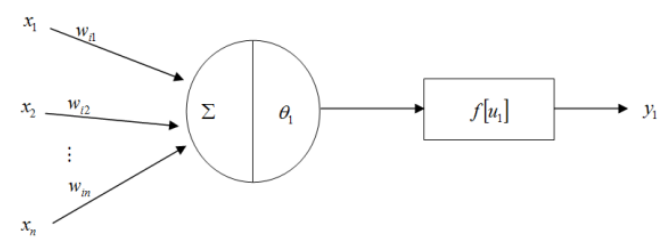

Figure 5.Schematic of mathematical models of neurons

Symbol description in Figure 5 is shown in Table 1:

Table 1. Symbol description in the mathematical model

\begin{tabular}{ll}
\hline Symbol & $\begin{array}{l}\text { Meaning of the } \\
\text { description }\end{array}$ \\
\hline$x_{1}, x_{2}, \cdots, x_{n}$ & $\begin{array}{l}\text { Input section of the } \\
\text { neuron, that is, }\end{array}$ \\
\hline
\end{tabular}




\begin{tabular}{ll}
\hline & information sent by the \\
& upper level \\
$\theta_{i}$ & $\begin{array}{l}\text { Threshold value of the } \\
\text { neuron }\end{array}$ \\
$y_{i}$ & Output of the neuron \\
$f\left[u_{1}\right]$ & Excitation function
\end{tabular}

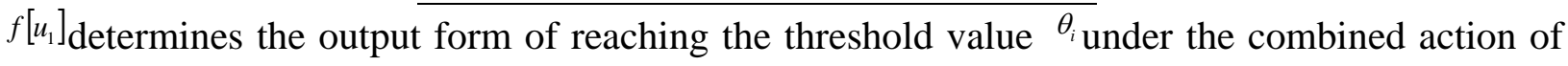
input $x_{1}, x_{2}, \cdots, x_{n}$. Figure 6 shows the images of two types of excitation function. The model used in this paper adopts the second type of the excitation function.
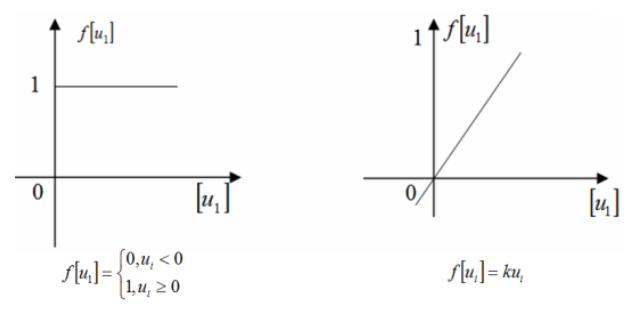

Figure 6. Typical excitation functions

$$
\text { Where, } u_{i}=\sum_{j} w_{i j} x_{i}-\theta_{i}
$$

So $y_{i}=f\left[u_{i}\right]=f\left(\sum_{j} w_{i j}-\theta_{i}\right)$

Formula (2) is a full expression of the mathematical models of a single neuron.

$B P$ neural network is a multi-layer feedforward network, which adopts the calculation method of the minimum mean square error.When the back-propagation algorithm is applied to the multi-layer feedforward network, Sigmoid is used as an excitation function, and the following steps are used for recursive calculation of $w_{i j}$, that is, the network weight coefficient. When each layer has $n_{\text {neurons, }}$ for the $i_{\text {neuron at the }} k$ layer, there are ${ }^{n}$ weight coefficients, namely, ${ }^{w_{i},}, w_{i 2}, \cdots, w_{j n}$. In addition, ${ }^{w_{n n+1}}$ is selected to represent ${ }^{\theta_{i}}$. When the sample ${ }^{x}$ is input, $x=\left(x_{1}, x_{2}, \cdots, x_{n}, 1\right)$.

1) Give a valuation to ${ }^{w_{i j}}$. Give a relatively small non-zero random number at each layer of ${ }^{w_{i j}}$, and $w_{j n+1}=-\theta_{i}$. This model operates by the use of Matlab, so the valuation process is a random process of the computer. Just because of this, if the same procedure code is in different operational processes, the results may be different.

2) Input the sample value $x_{x}\left(x_{1}, x_{2}, \cdots, x_{n}, 1\right)$, and the corresponding desired output $y=\left(y_{1}, y_{2}, \cdots, y_{n}, 1\right)$.

3) Calculate the output at each layer. For the output of the $i$ neuron at the $k$ layer, then:

$$
y_{i}^{k}=f\left[u_{i}^{k}\right]^{(3)}
$$

Where, $u_{i}^{k}=\sum_{j} w_{i j} x_{j}^{k-1}-\theta_{i}^{k}(4)$

Where, $x_{n+1}^{k-1}=1, \quad w_{i(n+1)}=-\theta$

4) Solve the calculation error $d_{i}^{k}$ at each layer. For the output layer, $k=m$, then:

$$
\begin{aligned}
& d_{i}^{m}=x_{i}^{m}\left(1-x_{i}^{m}\right)\left(x_{i}^{m}-y_{i}^{m}\right)(5) \\
& d_{i}^{k}=x_{i}^{k}\left(1-x_{i}^{k}\right)\left(\sum_{j} w_{i j} x_{j}^{k-1}-\theta_{i}^{k}\right)(6)
\end{aligned}
$$

5) Correct $w_{i j}$ and $\theta_{i}$, then:

$$
w_{i j}(t+1)=w_{i j}(t)-\eta d_{i}^{k} x_{j}^{k-1}(7)
$$

6) Determine whether it is consistent with the requirements according to the established 
standards after solving each weight coefficient at each layer. If not, then return to the third step, otherwise finish calculation.

The above calculation process is calculated by using Matlab programming, thus obtaining the distribution diagram about the eigenvalues of two different digital display platforms, as shown in Figure 7:

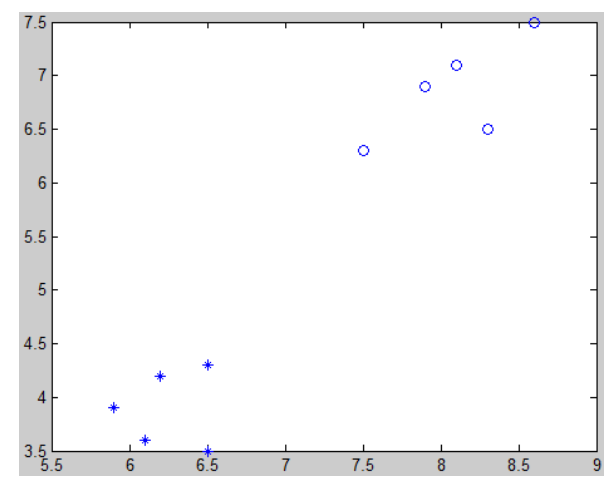

Figure 7. Two different virtual systems distribution of eigenvalues

In Figure 7, “*” represents the poor digital display platform, and “o” represents the excellent digital display platform. As can be seen from Figure 5, there are obvious limits in two different virtual systems.

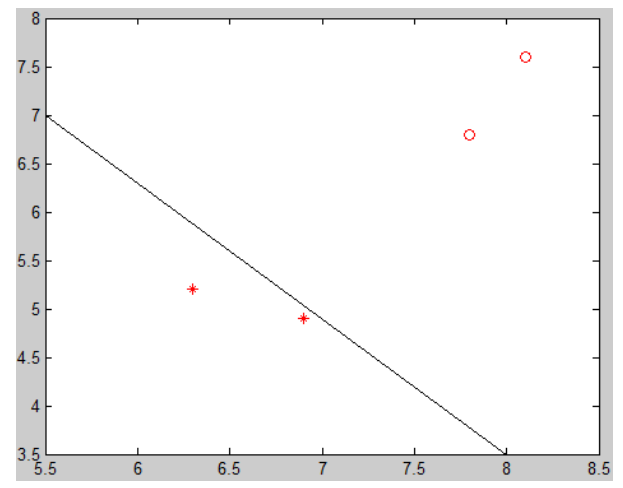

Figure 8.Evaluation results of the object being studied

Figure 8 shows the evaluation results of the object being studied. As can be seen from Figure 6, the effect of the digital display platform in Shandong Yikang Elderly Health Industrial Park and Zichuan Shanshuiyuan Community is better, while the effect of the digital display platform in Shijiazhuang Office Building and Wuzhen Town scenic spot is worse.

\section{Conclusion}

The virtual reality technology is the product of the rapid development of the information technology. In the process of real life, there are many factors limiting viewing. For example, the white-collar has a relatively large demand on the house purchasing, but the time to choose house is limited, which is not conducive to house purchasing. Applying the virtual reality technology to the digital display platform is in line with the people's living demands and conforms to the trend of society. Nowadays, the virtual reality technology can basically meet the requirements of the people, but the artistic sense of its digital display is not strong. It focuses on the technicality of the digital display but fails to give a visual impact. This paper adopts the BP neural network to establish a theoretical model of evaluating the effect of the virtual reality technology on the digital display platform, which is able to scientifically and visually determine the application of the virtual reality technology in the digital display design from the perspective of the mathematics. 


\section{References}

[1] Yang Wenjuan, et al. 2012. Empirical research on the virtual tourism quality evaluation base on the experience. Journal of Chongqing Normal University, (1): 100-104.

[2] Chen Xiaoying. 2013. Functional design and implementation of virtual tourism system - a case study of 3D electronic map of Lama Temple. Master's Thesis of Capital Normal University.

[3] Bi Yanna. 2012. Development research on virtual tourism products of intangible cultural heritage. Master's Thesis of Qingdao University,

[4] Song Yongpeng. 2012. Park virtual tourism system research and application - a case study of Longting Park. Master's Thesis of Henan University.

[5] Zhou Yongzheng, et al. 2010. Mathematical modeling. Shanghai: Tongji University Press,

[6] Wan Xinghuo. 2007. Probability Theory and Mathematical Statistics. Beijing: Science Press.

[7] Wang Xiaoyin, et al. 2010. Mathematical modeling and mathematical experiments. Beijing: Science Press.

[8] Zhuo Jinwu, et al. 2010. Application of Matlab in mathematical modeling. Beijing: Beijing University of Aeronautics and Astronautics Press.

[9] Li Sumin. 2008.Applied research of virtual reality technology in architecture and urban planning. Master's Thesis of Changsha University of Science and Technology,

[10] Xue Lei. 2007. 3D display and virtual immersion system based on Internet ancient buildings (Xiamen South Putuo Temple). Master's Thesis of Xiamen University,

[11] Jiang Huixian,\&Lin Guangfa. 2005. Discussion of Internet-based virtual tourism application. Journal of Southeast University (Philosophy and Social Science), 7 (Supplement): 216. 